\title{
Observación de anélides por el método de Río Hortega, modificado
}

\section{(Annellides staining by Río Hortega method modified)}

\author{
Luis Zaror C C 2* y Julieta T González $^{1}$ \\ ${ }^{1}$ Instituto de Microbiología Clínica, Facultad de Medicina, \\ Universidad Austral de Chile. Valdivia, Chile. \\ ${ }^{2}$ Escuela de Tecnología Médica, Universidad Mayor, sede Temuco.
}

*Autor para correspondencia: 1zaror@yahoo.es

RECIBIDO: 01 de Abril de 2019

APROBADO: 09 de Mayo de 2019

DOI: 10.22370/bolmicol.2019.34.1.1550

LOS AUTORES DECLARAN NO TENER CONFLICTO DE INTERESES

Palabras claves: Anélide; Identificación; Método de Río Hortega; Microndas

Key words: Annellide; identification; microwave: Rio Hortega Method.

\section{RESUMEN}

Es conocido ampliamente el uso de la conidiogénesis en la taxonomía de los hongos anamórficos. Una zona anelídica, puede ser indistinguible por esta metodología, causando confusión en la aplicación del término "anelídico" o "fialídico".

Se presenta una técnica basada en la combinación de la acción de microondas con el método de impregnación de argéntica de Río Hortega, modificado por Piva y colaboradores para dermopatología, aplicado a hongos anelídicos. Es de bajo costo, rápida, para demostrar los hongos anelídicos, en forma segura y reproducible, con un alto grado de definición en los detalles, en microscopio óptico de campo claro. El método de impregnación argéntica, usando un horno microondas doméstico durante 30 segundos, aplicado a ocho especies de hongos anelídicos, tuvo un por- centaje de éxito promedio de un 61\%, lográndose una técnica más corta con un alto grado de definición de las zonas aneladas. El promedio sube al 97\% al no considerar las especies de Exophiala. Los mejores resultados se obtuvieron con Scopulariopsis, Scedosporium y Hortaea. No se logró éxito en la observación de anelación en especies de Exophiala.

\section{ABSTRACT}

The use of conidiogenesis in the taxonomy of anamorphic fungi is widely known. An anellid zone may be indistinguishable by light field optical microscope, causing confusion in the application of the term "anellidic" or "phialidic".

A technique based on the combination of the action of microwaves with the method of impregnation of silver from Rio Hortega, modified 
by Piva and collaborators, applied to anellidic fungi is presented. It is of low cost, fast, to demonstrate the anellidic fungi, in a safe and reproducible way, with a high degree of definition in the details, in light field optical microscope. The silver impregnation method, using a domestic microwave oven for 30 seconds, applied to eight species of anellid fungi, had an average success rate of $61 \%$, achieving a shorter technique with a high degree of definition of the anellated zones. The average rises to $97 \%$ by not considering the Exophiala species. The best results were obtained with Scopulariopsis, Scedosporium and Hortaea. Success in observing anelation in Exophiala species was not achieved.

\section{INTRODUCCIÓN}

Los hongos filamentosos, son identificados por sus estructuras asexuales especializadas, para el reconocimiento de género y especie ${ }^{1}$. Salvo excepciones, no existen buenos métodos para observar anélides, requiriéndose para ello de microscopia de interferencia de fases o microscopia electrónica. Los hongos anelídicos son muy heterogéneos y evocan las fiálides en la producción de conidios, en cadenas basípetas y ello puede llevar a errores de identificación.

Kendrick (1971)², define la anélide como una forma especializada de ontogenia holoblástica en cuyo desarrollo se forman sucesivas proliferaciones percurrentes de la célula conidiógena (usualmente más larga), dando origen a una sucesión basípeta de conidios por anelación de la anélide. El conidio anelídico se reconoce indirectamente, por su base trunca, donde en cada anelación un nuevo trozo de material de pared es depositado en cubiertas internas ${ }^{3}$. Las anelaciones no siempre son visibles al microscopio óptico, especialmente cuando la célula esporógena es hialina ${ }^{4}$. A veces, solo pequeñas irregularidades permiten la orientación hacia la identificación del aneloconi- dio y anélide. Piva y colaboradores ${ }^{5}$, combinaron la acción del microondas con uno de los métodos de tinción del carbonato de plata amoniacal de Río Hortega, en dermatohistopatología, acortando el tiempo empleado en la técnica original, con un alto grado de definición en las imágenes, para aplicarla a la observación de hongos en cortes de tejido, generando la posibilidad de contar con un método que permita observarlas de forma rápida, con resultados constantes, y sin utilizar equipo costoso $(1999,2001)^{5,6}$. A partir de las observaciones anteriores, se presenta el método de Río Hortega, modificado por Piva y colaboradores, como técnica para la visualización de anélides.

\section{MATERIALES Y MÉTODO}

La preparación del cepario de hongos anelídicos en estudio, se realizó en agar Sabouraud dextrosa y agar Lactrimel, por incubación durante 7-15 días a $28^{\circ} \mathrm{C}$. A partir de estos medios se desarrollaron microcultivos, a los que se les aplicó la coloración de Río Hortega modificada por Piva y colaboradores ${ }^{6}$ y se observaron con microscopia óptica. Se trabajó once cepas de ocho especies de hongos anelídicos (tabla 1).

Técnica inicial: En una placa Petri con herradura de vidrio, porta objetos y 2 a 3 cubreobjetos, estériles, poner asépticamente, tres trozos cuadrados de agar Lactrimel de 0.6 x $0.6 \mathrm{~mm}$. Con gancho estéril, inocular el hongo en estudio, en los trozos de agar y cubrir con cubreobjeto estéril. Adicionar 5-10 $\mathrm{ml}$ de suero fisiológico estéril. Cerrar la placa e incubar a $28^{\circ} \mathrm{C}$ por cinco días mínimo (según velocidad de crecimiento del hongo). Transcurrido este período, retirar cuidadosamente el cubreobjeto, en el cual se aplica la tinción argéntica en estudio. Se utilizaron para la tinción microcultivos de siete, quince, treinta y sesenta días de incubación (tabla 2), según el desarrollo del hongo en estudio, buscando el mejor tiempo de observación de la anélide. 
Tabla 1. Hongos anelídicos estudiados, para observación de anélides.

\begin{tabular}{|l|l|l|}
\hline$N^{\circ}$ & ESPECIE & N \\
\hline 1 & Scopulariopsis brevicaulis. & $24,7940,8616,8623$ \\
\hline 2 & Scopulariopsis brumptii. & FMR 183 \\
\hline 3 & Scedosporium apiospermum. & 1105 \\
\hline 4 & Scedosporium prolificans $*$ & 229 \\
\hline 5 & Exophiala dermatitidis. & 2 \\
\hline 6 & Exophiala jeanselmei & FMR 3 \\
\hline 7 & Exophiala salmonis. & 419 \\
\hline 8 & Hortaea werneckii. & 14 \\
\hline
\end{tabular}

Tabla 2. Estandarización de días de crecimiento de microcultivos, para la visualización de anélide. Método de Río Hortega, modificado.

\begin{tabular}{|c|c|c|c|c|c|}
\hline Especies & $7 \mathrm{~d}$ & $10 \mathrm{~d}$ & $15 \mathrm{~d}$ & $30 \mathrm{~d}$ & $60 \mathrm{~d}$ \\
\hline Scopulariopsis brevicaulis & + & + & ++ & ++ & ++ \\
\hline Scopulariopsis brumptii & - & + & ++ & ++ & ++ \\
\hline Scedosporium apiospermum & - & - & - & - & + \\
\hline Scedosporium prolificans & - & - & - & - & + \\
\hline Exophiala dermatitidis & - & - & - & - & - \\
\hline Exophiala jeanselmei & - & - & - & - & - \\
\hline Exophiala salmonis & - & - & - & - & - \\
\hline Hortaea werneckii & - & - & + & + & + \\
\hline
\end{tabular}

Resultados se expresan en cruces, siendo mejores a más días de incubación. Observación de anélide: (++) adecuada; (+) moderada; (-) no se observa.

\section{Preparación de los reactivos:}

Reactivo de Río Hortega: A $30 \mathrm{ml}$ de nitrato de plata al $10 \%$, agregar $90 \mathrm{ml}$ de carbonato de sodio al $5 \%$. Se forma un precipitado, que se disuelve agregando amoniaco al 28\%, gota a gota. Completar con agua destilada hasta $400 \mathrm{ml}$, conservándose en frasco ámbar. Formol al 1\%, Tiosulfato de sodio al $5 \%$.

\section{DESARROLLO DE LA TÉCNICA.}

Para estandarizar la técnica, aplicable a los hongos anelídicos, se consideraron varios parámetros: nivel de potencia del horno microondas (nivel bajo, medio, medio alto y alto), tiempo de coloración con el reactivo de Río Hortega en el horno microondas (12, 15, 30 y 40 segundos), tiempo de reducción de la plata en formalina $(15,30,60 \mathrm{y}$ 
120 segundos), y tiempo de fijación en tiosulfato (30, 45 y 60 segundos).

1.-Tomar el cubreobjetos, del microcultivo, con el hongo anelídico adherido y flamear tres veces (1 segundo cada vez).

2.- Sumergir el cubreobjetos en el reactivo de Río Hortega, modificado por Piva y col.

3.-Poner, en horno de microondas (modelo Daenix-car 701, 375 w. y 2450 Mhz.) en un nivel de potencia (bajo, medio, medio alto y alto) y tiempo $(12,15,30$ y 40 segundos) seleccionando y alternando los parámetros en estudio.

4.-Realizar reducción de la plata sumergiendo el cubreobjetos en formol al 1\%, por 15, 30, 60 y 120 segundos, (agitando o soplando el preparado).

5.-Repetir el paso 3 y 4, si el espejo de plata no se ha formado.

6.-Sumergir el cubreobjetos en tiosulfato de sodio al 5\%, durante 30, 45 o 60 segundos, para fijar la preparación.

7.- Lavar, sumergiendo la preparación en un vaso de precipitado con agua destilada. Secar, cuidadosamente de modo de no desprender el hongo.

8.-Hacer un cuadrado con esmalte de uñas, depositar una o dos gotas de lactofenol con o sin tinta Parker 51 azul permanente, colocar el cubreobjeto sobre portaobjetos y sellar la preparación.

Se procesaron en el estudio 1013 microcultivos (tabla 4).

Las fotografías fueron realizadas en un microscopio binocular Olympus BHT-2, con cámara fotográfica Samsung Digmac- 210, de 2.1 megapixels.

\section{RESULTADOS}

Los resultados en algunos parámetros variaron según la especie y otros se mantuvieron para todos iguales, como lo demuestran las tablas 2, 3,4 y las descripciones pertinentes. El tiempo de crecimiento de los microcultivos según el "método de Riddel" (Riddel, 1950), para colorear y la posterior observación de anélides, se estandarizó según se muestra en la tabla 2, obteniéndose los mejores logros en Scopulariopsis brevicaulis, S. brumptii y Hortaea werneckii a los 15 días de incubación. En Scedosporium apiospermum y S. prolificans los mejores resultados fueron obtenidos a los 60 días de incubación. Con Exophiala sp., aún a los 60 días de estudio no se observó anélide.

El nivel de potencia con que se obtuvo mejores resultados fue el nivel medio en todas las especies de hongos utilizadas. El mejor tiempo, entre 12 a 40 segundos, para teñir las láminas con el reactivo de Río Hortega en microondas, fue de 30 segundos en todas las especies de hongos estudiadas. El tiempo en que las láminas fueron reducidas en formalina al 1\% se estandarizó con mejores resultados a los 120 segundos, momento en el cual se aprecia la formación de un espejo de plata en la lámina. El mejor tiempo de fijación con el tiosulfato de sodio al 5\%, se logró a los 45 segundos.

Para cada hongo se consideró variaciones propias de las características morfológicas y de crecimiento, sin embargo, en el desarrollo de la técnica se unificaron los resultados, utilizando un único protocolo.

\section{Protocolo para hongos anelídicos, método de Río Hortega, modificado:}

- A partir de un microcultivo de un hongo anelídico, tomar el cubreobjeto y flamear tres veces (1 segundo cada vez). 
- Aplicar la coloración con el reactivo de Río Hortega (del carbonato de plata amoniacal), modificado por Piva y col. Para ello, sumergir el cubreobjetos en una placa Petri con el reactivo.

- Poner, luego en horno de microondas durante 30 segundos a $375 \mathrm{~W}$.

- Realizar reducción sumergiendo el cubreobjetos en formol al 1\%, por 1 minuto (agitando o soplando el preparado).

- Repetir el paso 3 y 4, si el espejo de plata no se ha formado.

- Sumergir el cubreobjetos en tiosulfato de sodio al 5\%, durante 45 segundos, para fijar la preparación.

- Lavar, sumergiendo la preparación en un vaso de precipitado con agua destilada. Secar, cuidadosamente para no desprender el hongo.

- Colocar el cubreobjeto sobre portaobjetos, realizando previamente un cuadrado con esmalte de uñas, y adicionar lactofenol con o sin tinta o chloro-black E. Sellar la preparación con esmalte de uñas. Observar.

En total, se realizaron 1013 láminas, a las cuales se les aplicó la coloración argéntica, observándose según muestra la tabla 4 , que en los géneros Scopulariopsis, Hortaea y Scedosporium se logró los mejores resultados expresados en porcentaje de éxito, con observación clara y bien definida de las anélides (figura 1). En Exophiala, en muchas de las láminas realizadas se logró la observación de esbozos de las anélides, sin embargo, se consideró un $0 \%$ de éxito, debido a que no estaban claras ni definidas.

Tabla 3. Estandarización de nivel de potencia en horno microondas, para la aplicación del reactivo de Río Hortega, modificado.

\begin{tabular}{|l|l|l|l|l|}
\hline \multicolumn{1}{|c|}{ Especies } & Bajo & Medio & $\begin{array}{l}\text { Medio } \\
\text { alto }\end{array}$ & Alto \\
\hline Scopulariopsis brevicaulis & - & ++ & $+/-$ & - \\
\hline Scopulariopsis brumptii & - & ++ & +/- & - \\
\hline Scedosporium apiospermum & - & + & - & - \\
\hline Scedosporium prolificans & - & + & - & - \\
\hline Exophiala dermatitidis & - & $+/-$ & - & - \\
\hline Exophialajeanselmei & - & $+/-$ & - & - \\
\hline Exophiala salmonis & - & $+/-$ & - & - \\
\hline Hortaea werneckii & - & ++ & $+/-$ & - \\
\hline
\end{tabular}

Los resultados están expresados en cruces, obteniéndose mejores logros en el nivel medio. Intensidad de impregnación del reactivo de Río Hortega, modificado: $(++)$ intensa sin precipitados; $(+)$ moderada sin precipitados; $(+/-)$ leve sin precipitados; $(-)$ no se impregna o se forman precipitados inespecíficos de plata por exceso de calor. 
Tabla 4. Resumen de láminas realizadas y porcentaje de éxito para la observación de anélides.

\begin{tabular}{|l|l|l|}
\hline Especies & cantidad & \% de éxito \\
\hline Scopulariopsis brevicaulis & 450 & 98 \\
\hline Scopulariopsis brumptii & 120 & 97 \\
\hline Scedosporium apiospermum & 75 & 95 \\
\hline Scedosporium prolificans $*$ & 108 & 96 \\
\hline Exophiala dermatitidis & 65 & 0 \\
\hline Exophialajeanselmei & 65 & 0 \\
\hline Exophiala salmonis & 65 & 0 \\
\hline Hortaea werneckii & 65 & 98 \\
\hline Total cantidad/ promedio \% & 1013 & 61 \\
\hline
\end{tabular}

\section{- Lomentospora}
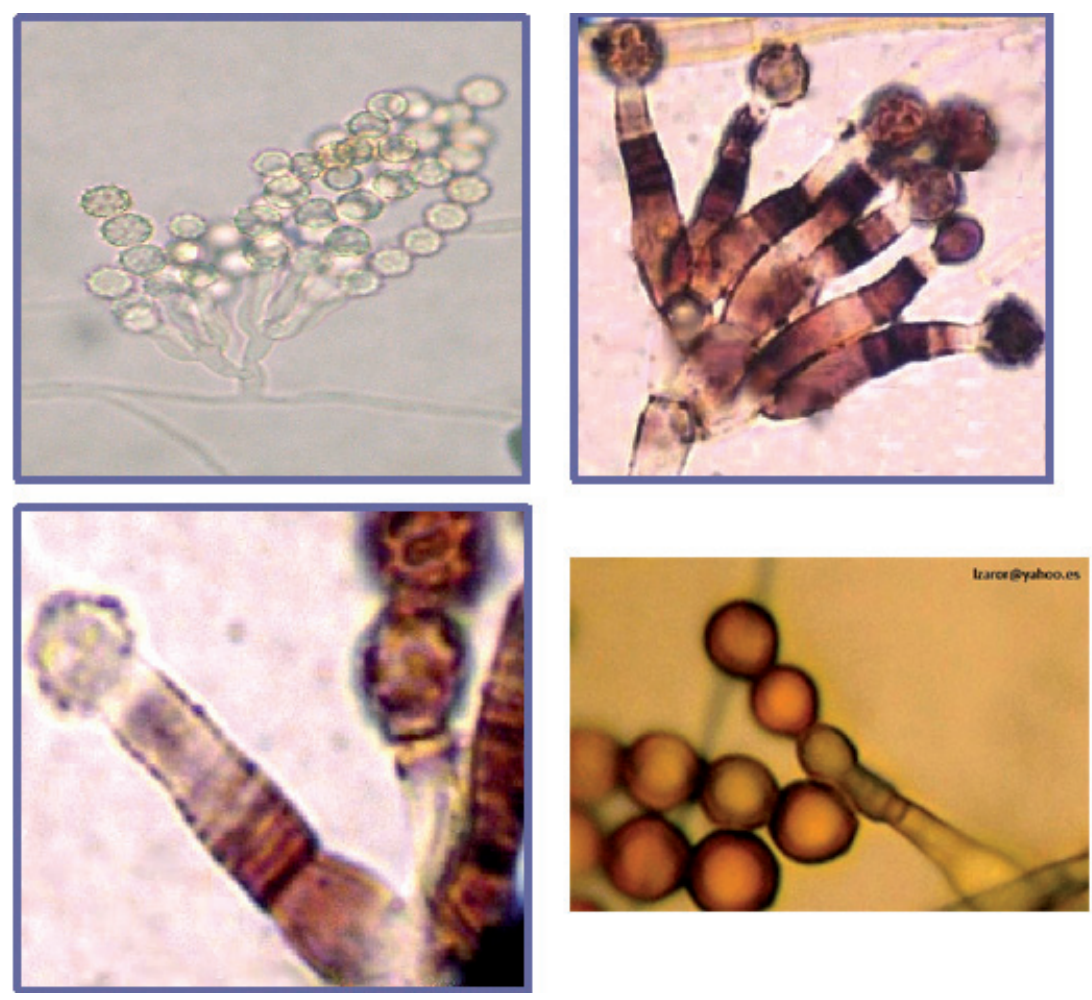

Figura 1. a) Observación de Scopulariopsis brevicaulis, con microscopía de campo claro con lactofenol sin tinta. No se observa anélide. (Fig.1a. x1000). b) y c) observación de anélides y anillos cicatrizales de Scopulariopsis brevicaulis, a partir de microcultivos con coloración de Río Hortega, modificado por Piva y colaboradores y montada con lactofenol con tinta. (Fig. 1b., 1c. x1000). d) Scopulariopsis brumptii, anélide y anillos cicatrizales. 

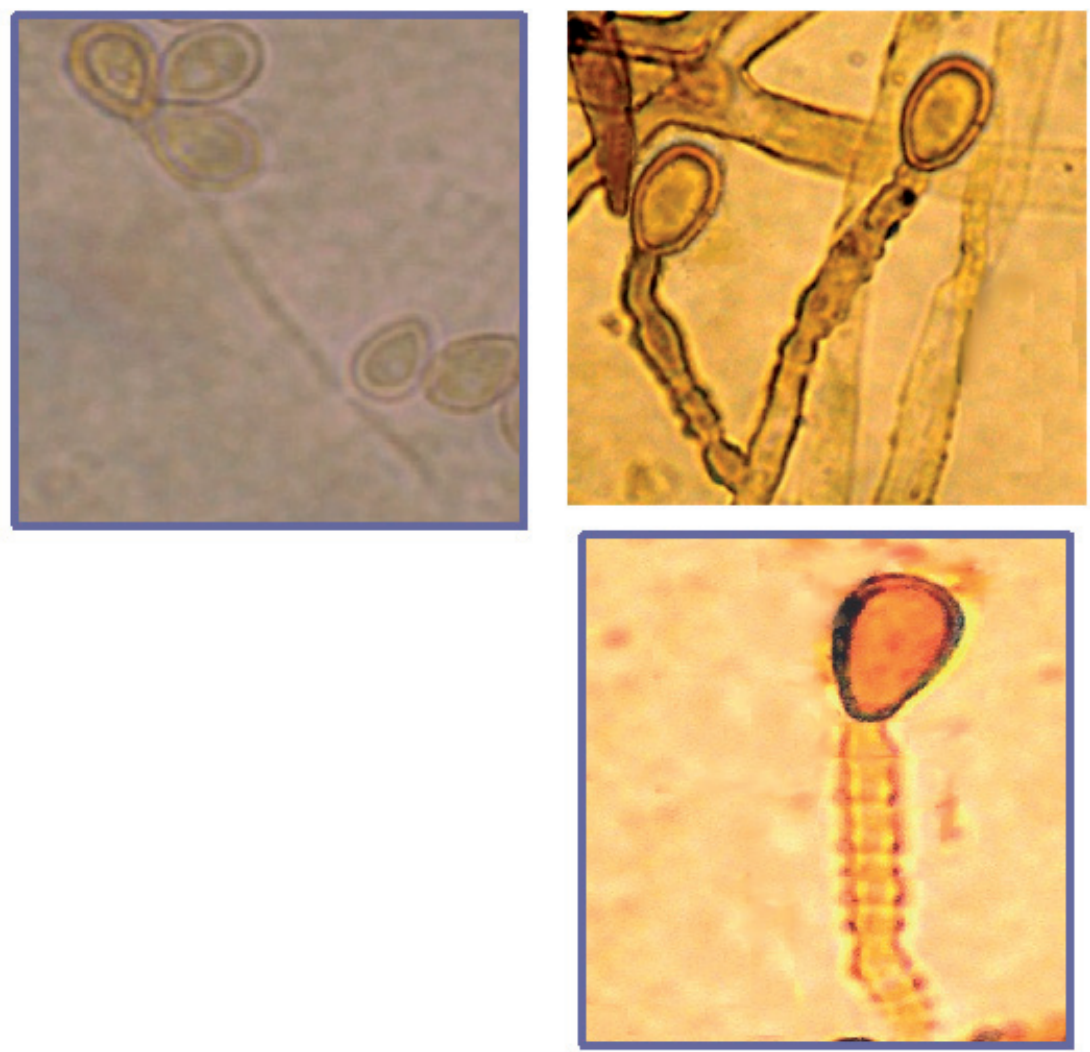

Figura 2. Scedosporium apiospermun, a) microfotografía de microscopia de campo claro, preparación con lactofenol-tinta. No se observan anélides. (x1000). b) se observan depósitos de pared cicatrizales y anélides, mientras en c) anélides bien visibles después de 60 días de incubación, teñidas con coloración de Río Hortega, modificada por Piva y colaboradores. (x1000).

\section{DISCUSIÓN}

La terminología para denominar la conidiogénesis y su aplicación taxonómica en hongos anamórficos está basada en la observación de sus estructuras en microscopía de campo claro, lo que crea ambigüedad e imprecisión por la dificultad de observar y establecer si se está en presencia de un hongo anelídico o fialídico?

Mediante microscopía electrónica se ha logrado obtener una identificación más certera; sin embargo; esto implica contar con equipamiento sofisticado ${ }^{4}$. Otra causa de ambigüedad, es el descubrimiento de muchas excepciones en el concepto básico de tipos de conidiogénesis. Un caso particular es Hortaea werneckii, que forma conidios sub- secuentes, por una parte, simpodialmente, además de producir conidios percurrentes, que conducen a una confusión en la aplicación de los términos "anelídico" o "fialídico"?.

Este estudio permite contribuir, a observar anillos de las anélides en el grupo de hongos en estudio; no siempre visibles en microscopio óptico, especialmente cuando la célula esporógena es hialina; mediante la técnica de tinción de Río Hortega, modificada, por Piva y colaboradores ${ }^{5,6}$ con microscopia de campo claro (fig 1,2). La técnica de impregnación argéntica, combinada con la acción de microondas, permite obtener la definición morfológica de anélides y sus anillos en hongos, de forma rápida, eficiente, con resultados constantes, preparaciones permanentes, sin la utilización 
de equipamiento costoso, trabajar con un reactivo estable y duradero. La técnica modificada, no usa piridina $^{8}$ con actividad carcinogénica ${ }^{6}$.

Los hongos tienen en sus membranas y pared, abundantes carbohidratos, proteínas, lípidos, sales inorgánicas y pigmentos, con variaciones en cada género ${ }^{9}$, lo que permite que casi todas las estructuras fúngicas sean teñidas con la plata, logrando una excelente observación histológica ${ }^{6,10}$.

La irradiación de microondas durante la impregnación argéntica produce buenos resultados, en especial al ser aplicada por 30 segundos y en el nivel medio de potencia, según el selector del horno utilizado. Aplicando microondas se tiene la posibilidad de ampliar su uso a la rutina de trabajo en la identificación y diagnóstico de hongos. Al usar horno microondas, debe cuidarse que la temperatura no supere $\operatorname{los} 50^{\circ} \mathrm{C}$, debido a la formación de precipitados de plata.

Se realizaron 1013 láminas de microcultivo de los hongos en estudio (Tabla 4) aplicándoles la técnica de impregnación argéntica con un promedio de porcentaje de éxito, para la visualización de anélides de un 61\%. Sin embargo; el porcentaje de éxito en la observación de las anélides en Scopulariopsis brevicaulis, $S$ brumptii, Hortaea werneckii, Scedosporium apiospermun y S. prolificans fue alrededor de un 97\%. En las especies de Exophia$l a$, el porcentaje de éxito, en la observación de anélides fue de $0 \%$.
Los géneros Scopulariopsis y Hortaea, son descritos con zona anelídica amplia, lo que permitió que en microcultivos de 15 días fuera aplicada la coloración argéntica exitosamente, observándose una clara correlación entre las estructuras definidas por otros investigadores, con la ayuda de estudios mediante microscopia electrónica ${ }^{4,6,11}$ y la técnica de impregnación argéntica. La anélide y anillos fueron observados de color café a negruzco.

El género Scedosporium, presenta una zona anelada inconspicua ${ }^{11}$, sin embargo, se utilizó microcultivo de 60 días para aplicar la coloración argéntica, lo que permitió, a nuestro juicio, el engrosamiento suficiente de los anillos de la pared, para su visualización (figura 2).

El género Exophiala es descrito con zonas anelídicas cortas, finas e inconspicuas, además, de su naturaleza polimórfica al presentar más de un estado conidial, lo que ha originado siempre problemas en la clasificación taxonómica ${ }^{4,11}$. En nuestro estudio, esta particularidad del género también nos afectó negativamente, no lográndose la observación de la anélide. Según nuestras observaciones, el 0\% de éxito, en realidad sólo refleja que no se visualizaron los anillos cicatrizales en forma clara y bien definida; sin embargo, se observaron esbozos de éstos. Tal vez, el uso de medios de cultivo que estimulen la conidiogénesis o de reactivos que aumenten el grosor de los anillos, posibilite la mejor observación de estas estructuras, en géneros donde es difícil lograr resultados, como es el caso de Exophiala.

\section{REFERENCIAS}

1. St-Germain, G. \& Summerbell, R. Identifying Filamentous Fungi. Star Publishing Company. Belmont. 1996

2. Kendrick, W.B. Taxonomy of Fungi Imperfecti. Univ. Toronto Press, Toronto. 1971.

3. Hughes, S.J. Conidiophores, conidia and classi- fication. Can. Journal Bot. 195, 131: 577-659.

4. Piontelli, E. y Toro, M.A. Hyphomycetes. Conceptos antiguos y modernos de su clasificación. Análisis crítico desde el punto de vista del micólogo médico. Boletín del Instituto de Salud Pública de Chile. 1978, 21(1):42-73. 
5. Piva, J.R., Piva, C.E., Ortega, H.H., Salvetti, N.R. Microwave-enhanced of a method of silver impregnation applied in dermatohistopathology. Biocell. 1999, 23(2):143-147.

6. Piva, J.R., Ortega, H.H., Canal, A.M., Piva, C.E., Reus, V., Seib, E.P. Identificación de hongos en tejido y cultivos: Importancia de sustancias argirófilas en su estructura. Rev. Fac. Cienc. Med. Córdoba. 2001, 58(1):57-64.

7. De Hoog S, Torres Rodriguez, J.M. Proceedings $\mathrm{X}$. Congress of the international society for human and animal mycology. Masson S.A., Barcelona, España.1988.
8. Lynn, L.H., Mcreight, J.D. and Christie, R.D. Microwave enhanced staining for plant virus inclusions.. Biotechnic and Histochemistry. 1992, 67(1):40-44.

9. Alexopoulos, C.J., Mins, C.W., Blackwell, M. Introductory Mycology. 4ta Ed. John Wiley \& Sons, Inc. USA. 1996.

10. Ganter, P, Jolles G. Histochimie, Normale et Pathologique. Gauther Williams, 2da Ed. Canadá. 1969.

11. De Hoog, G.S. \& Guarro, J. Atlas of Clinical Fungi. CBS. Utrecht/Universitat Rovira iVirgili, Reus, 2000. Ed. 2da., España. 